\title{
La formación interdisciplinaria de los profesores: una necesidad del proceso de enseñanza y aprendizaje de las ciencias
}

Giselle León León

La educación contemporánea debe caracterizarse en el quehacer pedagógico por su estructura curricular, por su desarrollo metodológico e integración de los contenidos (conocimientos, habilidades, actitudes y valores) y por experiencias que faciliten una comprensión más reflexiva y crítica de la realidad. De allí, que se plantea la necesidad de una educación que forme a un individuo "no fragmentado" y lo prepare para una educación permanente. Una educación que revalorice, además, "sus aspectos éticos y culturales" (Delors J. 1996: 21), basada en cuatro pilares: "Aprender a conocer, aprender a actuar, aprender a vivir juntos y aprender a ser" (Delors J. 1996: 10).

En concordancia con la valoración teórica y la realidad actual, se hace necesario establecer relaciones entre las diferentes disciplinas cuando se van a analizar hechos de la vida diaria y también de la ciencia y de la tecnología. Esto se hizo cotidiano desde el momento mismo en que las áreas 
comenzaron su desarrollo independiente, por lo que un reclamo muy difundido es aquel que invoca a establecer la interdisciplinariedad.

Definir este término es algo difícil, se debe tener presente el criterio de diferentes autores, puesto que cada uno de ellos aporta algo en particular, pero en lo que la mayoría están de acuerdo es que, en esencia es una filosofía de trabajo que implica la colaboración de un colectivo de personas, en este caso de profesores y maestros, teniendo presente que cada uno de los que intervengan en esta labor común, tenga competencia en su disciplina y ciertos conocimientos de los contenidos y métodos de trabajo de las otras.

La interdisciplinariedad no puede ser espontánea, debe colegiarse entre todos los integrantes, para que sea considerada una estrategia de enseñanza aprendizaje, en la cual el centro sea el sujeto que aprende y para el colectivo pedagógico un método de trabajo de sus alumnos.

Es también un criterio que prevalece aquel que señala a la interdisciplinariedad como propiciadora de un cambio no sólo conceptual, sino también metodológico y actitudinal.

\section{¿Cómo se define a la interdisciplinariedad?}

Según Ander-Egg, (1999), evoca la idea de intercambio entre diferentes disciplinas con la meta de generar un conocimiento. Por su parte, el filósofo y político, francés Morin (1998), manifiesta que "es un requerimiento innovador dirigido a superar un saber fragmentado, es un intercambio y cooperación ordenada de disciplinas".

Miguel Fernández Pérez (1994), profesor español, señala que "la interdisciplinariedad no es un diseño de contenidos mezclados en un currículo, sino como objeto curricular es una manera de pensar, es un hábito de aproximación a la construcción de cualquier conocimiento, que al ser método didáctico, deviene método del alumno". 
A pesar de que el consenso general es concluyente a favor de establecer la interdisciplinariedad, es algo difícil de encontrar, ejemplificadas, las vías que permitan llevarla a cabo, es un desafío no exento de dificultades, existen factores que obstaculizan el desarrollo de este trabajo, los cuales se pueden resumir en:

- La concepción curricular de los planes de formación de profesores no favorece una concepción interdisciplinaria (formación disciplinar por un lado y la pedagógica por otro).

- Los docentes no poseen los conocimientos de otras disciplinas, que permitan la proyección y participación en proyectos interdisciplinarios.

- La predisposición de los docentes en las formas y vías de impartir la docencia.

- La no conceptualización del proceso de enseñanza aprendizaje como un proceso activo, dinámico, grupal e individual, social y contextualizado.

- El fundamento epistemológico, que permite el análisis de los fenómenos de la naturaleza y la sociedad como un todo.

En la esfera educacional, la interdisciplinariedad ha sido tratada más como cuestión teórica que llevada a la práctica. Ha sido declarada en los diseños curriculares y documentos metodológicos como intención, como aspiración, pero no se ha concretado en la práctica pedagógica mediante acciones específicas.

En nuestras condiciones es necesario tener en cuenta la importancia del colectivo pedagógico, del claustrillo para la puesta en práctica de nuestro proyecto educativo; en el que el trabajo interdisciplinario debe ser primordial.

Es definitivo, los formadores de formadores son los que deben originar el trabajo interdisciplinario, y pueden 
definir la interdisciplinariedad como una estrategia didáctica asumida por un grupo de docentes, para propiciar un aprendizaje holístico y significativo, en el caso de nuestro centro, estrategia didáctica asumida por un grupo de docentes, para propiciar la formación del futuro docente y su desempeño en un contexto educacional.

En ese colectivo deben cumplirse las siguientes condiciones:

- Cada docente debe ser competente en su disciplina y tener ciertos conocimientos.

- Debe tener una actitud abierta a nuevos métodos de abordaje de la realidad.

- No debe pensar que su criterio es el único y que su verdad es absoluta.

Los docentes de ese colectivo debemos comprender el proceso de enseñanza aprendizaje como un proceso dialéctico, institucional, dirigido, sistemático, bilateral y activo, de naturaleza social y grupal, contextual e individual. En el que se generen estrategias de enseñanza aprendizaje; sobre la base de la actividad y de la comunicación, docentes y alumnos construyen y reconstruyen el conocimiento, se practican los valores y se aprenden las conductas más deseables para la sociedad. A nivel individual el educando aprende lo que considere más adecuado para la sociedad, sin dejar de lado que esto se encuentra muy determinado por el contexto familiar y social en que se desenvuelve.

En el área de ciencias naturales o en otras disciplinas se necesita estructurar un colectivo que cumpla con las condiciones señaladas, donde los actores debaten aspectos conceptuales y metodológicos de cada disciplina particular y de la didáctica general, para propiciar la preparación del docente de tal manera que le permita enfrentar el trabajo 
interdisciplinario en el colectivo.

En cada centro educativo se debe estructurar el trabajo interdisciplinar, para propiciar la aplicación del principio de la interdisciplinariedad en cada clase, en cada asignatura, por cada miembro del colectivo, y por supuesto que el Centro de Investigación y Docencia en Educación, CIDE, de la Universidad Nacional, en su labor formadora de docentes no lo deje de lado en cada curso y cada grado, de tal forma que sea nuestra realidad.

Este pensamiento interdisciplinar y su concreción en cada clase debe favorecer que el alumno se enfrente a situaciones que han de ser resueltas aplicando dicha concepción, esto propiciará que el estudiante la asuma como método propio de trabajo, que debe favorecer la formación de hábitos intelectuales, la comprensión holística de la realidad, la posibilidad de intervención en todas las esferas de la vida y como diría Morin (1998), "ecologizar" las disciplinas, es decir, tomar en cuenta todo lo que es contextual comprendiendo las condiciones culturales y sociales, es decir, ver en que medio ellas nacen, plantean el problema, se esclerosan y se metamorfosean.

¿Qué vías podemos utilizar para realizar un proceso de enseñanza aprendizaje donde se aplique sistemáticamente la interdisciplinariedad?

La respuesta a esta interrogante debe salir del docente, en cada una de las actividades metodológicas y de otro tipo que se realicen.

Esta actividad implica un trabajo colectivo, en el que se deben determinar los nodos interdisciplinarios, la discusión en los colectivos de los conocimientos básicos tanto metodológicos como científicos de las asignaturas, con el propósito de que todos los docentes sean capaces en nuestras disciplinas y tengan conocimientos básicos de las otras (Ander-Egg, 1999). 
Una vía que puede ser utilizada, y que muchos docentes lo hacen es plantear al alumno situaciones de aprendizaje, que para ser resueltas impliquen un análisis interdisciplinario del fenómeno y a la vez una investigación por parte de los estudiantes, que propicien el desarrollo de las habilidades investigativas, de búsqueda bibliográfica, de confección de resúmenes, etc., habilidades que debe poseer todas las personas.

Ejemplos de estas situaciones pueden ser:

- ¿Por qué se prepara a los deportistas de alto rendimiento, durante determinado periodo de tiempo, en puntos geográficos de altura elevada?

- QQué procesos relacionados con el agua se producen en la naturaleza que dan origen a la formación de cuevas y de formas cársicas?

- ¿Por qué puede llegar a la retina el haz de luz, que nos permite la visión?

Todas estas situaciones de aprendizaje para ser resueltas por los educandos, conlleva un análisis interdisciplinario del fenómeno, que implica que el estudiante participe de su propio aprendizaje, por lo que debe ser significativo para él.

Otra vía que puede ser utilizada para realizar este trabajo es la realización de trabajos prácticos, en los que intervengan diferentes disciplinas y/o asignaturas, a partir de la concepción del trabajo del departamento y del colectivo de año, y que tiene una salida en trabajos relacionados con los ejes transversales como por ejemplo: educación para la cultura ambiental y educación para el respeto a toda forma de vida, en el que pueden intervenir la Química, la Geografía, la Biología, la Física, la Matemática, el Español y la Historia en un análisis holístico del problema. 
Esto permitirá a partir de la relación interdisciplinaria, lograr en los estudiantes, una mejor asimilación de los contenidos, y a generar en ellos una conciencia global de los contenidos. Es importante tener en cuenta además que el profesor como elemento orientador del proceso docente, es a la vez un investigador y debe adentrarse cada vez más en el estudio de los problemas referentes al aprendizaje de sus alumnos, para modificar sus métodos y los modelos de actuación. Esta será su labor cotidiana en el aula, lo que le permitirá lograr un proceso de enseñanza y aprendizaje dialéctico y novedoso.

Por esto, la interdisciplinariedad se convierte en una manera de pensar, un hábito que nos permite la aproximación a cualquier conocimiento y por lo tanto, es un principio didáctico que debe ser asumido por el alumno, en este caso del futuro profesor, que deberá emplear los métodos de enseñanza aprendizaje más acorde con las exigencias, que implica un trabajo interdisciplinario.

Se debe tener presente que se desea formar un profesor capacitado para realizar cambios a su práctica cotidiana, mejorar su docencia y que constantemente modifique su accionar, por ende debe tener cierta concepción acerca de la epistemología de las ciencias y de la investigación científica, situación que toma relevancia por cuanto hay un extraordinario desarrollo de la ciencia y la técnica a nivel mundial.

Estos precedentes nos indican que se debe realizar reformas curriculares, que propician el fortalecimiento de la calidad del estudiante y su capacitación en ciencias y tecnologías, pero también las investigaciones en pedagogía, que favorezcan el desarrollo de una práctica y una teoría de la enseñanza, acorde con los nuevos requerimientos, para que los formadores de formadores, se puedan preguntar: cómo llevar a la práctica pedagógica cotidiana la interdisciplinariedad como principio didáctico. 
La interdisciplinariedad, como principio didáctico, debe favorecer el desarrollo cognitivo del estudiante y brindarle un método de trabajo que propicie su desempeño profesional. Desde esta perspectiva a nivel pedagógico, lo que podemos hacer es enmarcarnos en la investigación dirigida. El análisis del desarrollo histórico de los diferentes paradigmas de aprendizaje nos ha llevado a determinar que una de las variantes que se utiliza actualmente para lograr un aprendizaje significativo es el de investigación dirigida, la cual tiene como base epistemológica el acercamiento que puede producirse entre el aprendizaje y la investigación científica; además de tomar en cuenta aspectos esenciales de ésta como son su contextualización (la relaciones ciencia- tecnología - sociedad) y los componentes afectivos (interés por la tarea, clima favorable para el trabajo) (Escudero,1990).

Esta variante de aprendizaje favorece la instrumentación de la interdisciplinariedad, pues potencia la participación del alumno en la búsqueda y adquisición del conocimiento y promueve la formación de un docente que debe tener como atributos:

- Conocer su disciplina con profundidad, sus leyes, teorías y conceptos fundamentales; así como los conceptos básicos esenciales de otras disciplinas que le permitan participar en proyectos interdisciplinarios.

- Haberse iniciado en la investigación de manera que le permita la comprensión de la metodología científica.

- Tener una cierta concepción epistemológica acerca de las ciencias, del método científico y de otras formas de conocimiento, para que su conocimiento científico disciplinar no sea neutral y absoluto, sino condicionado a la historia, a la sociedad y con una aplicación práctica.

- Establecer relaciones entre su disciplina y los problemas socioambientales, para que pueda proyectarse en ese aspecto. 
- Superar la idea de que los alumnos aprenden escuchando y memorizando lo que les da el profesor, como si no pudieran pensar y tener sus propias explicaciones sobre los hechos y fenómenos que se les presentan.

- Saber diseñar planes de actividades para favorecer la investigación de sus alumnos que deben ser:

- $\quad$ para acceder al pensamiento espontáneo de los alumnos y para motivarlos.

- para formular y definir en qué se va a investigar.

- para contrastar con las concepciones de los estudiantes.

- para aplicar el conocimiento adquirido en la investigación.

Partiendo de lo anteriormente expuesto, una estrategia de aprendizaje puede ser caracterizada por:

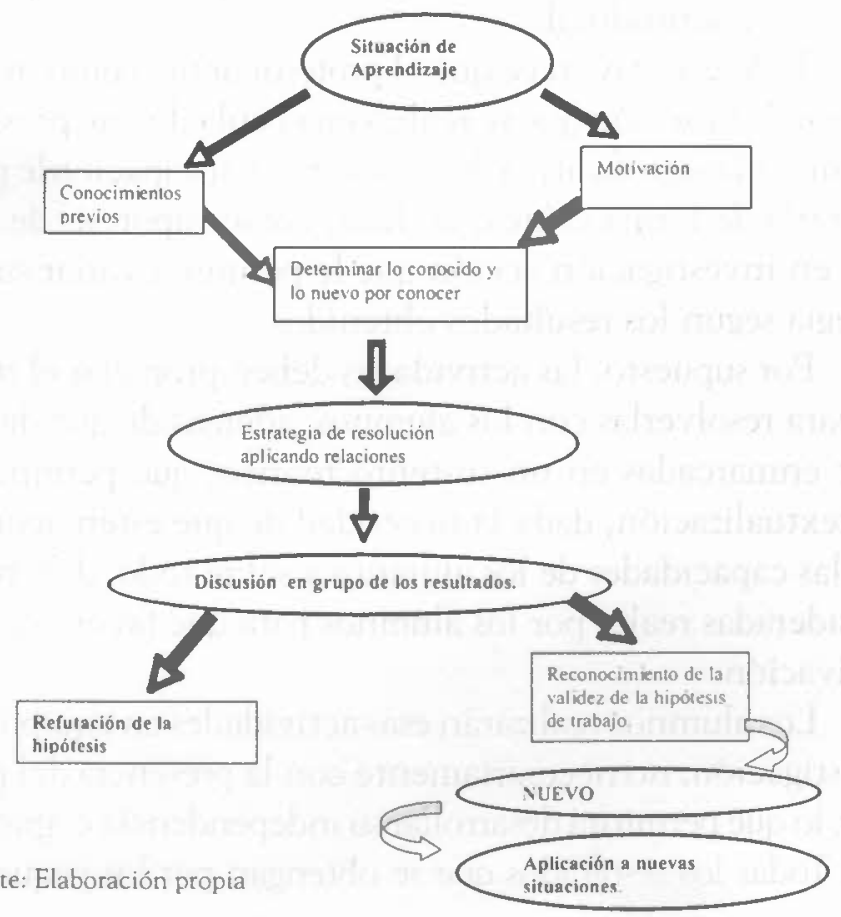


De tal manera, que se presentan a los alumnos situaciones de aprendizaje, que deben ser reales o muy próximas a la realidad, contextualizadas y que motiven al alumno a resolverlas. El proceso para su solución debe estar necesariamente basado en la aplicación de relaciones interdisciplinarias y en una investigación por parte de los alumnos.

Los alumnos con los conocimientos previos que poseen, analizan la situación planteada y determinan en qué aspectos deben profundizar para llegar a su solución, lo que implica un proceso de investigación, de formulación de hipótesis de trabajo o preguntas científicas que los guiarán. Se producirán conflictos cognitivos, no sólo en relación con los esquemas conceptuales sino también con los procedimientos o estrategias que se utilizan normalmente, así como los valores, las actitudes y normas asimiladas. De esta manera, se obtiene un cambio no sólo conceptual, sino metodológico y actitudinal.

Todo esto favorece que el profesor actúe como investigador de la acción que se realiza en el aula, lo que provoca una mejoría significativa de su docencia al capacitarle para valorarla de forma crítica, es decir, como hipótesis de trabajo en investigación acción que le permiten variar su estrategia según los resultados obtenidos.

Por supuesto, las actividades deben propiciar el interés para resolverlas con los alumnos, además de que deben estar enmarcadas en un sustento teórico, que permita su contextualización, dada la necesidad de que estén acordes con las capacidades de los alumnos y sobre todo, deben ser consideradas reales por los alumnos para que favorezcan la motivación.

Los alumnos realizarán esas actividades en equipos de investigación, no necesariamente con la presencia del profesor, lo que permitirá desarrollar su independencia cognoscitiva. Todas los resultados que se obtengan por los pequeños 
grupos de investigación deberán ser analizados en sesiones de debate, que propicien el intercambio científico, la discusión grupal, la obtención de un resultado integrado, que debe estar explicado sobre la base del sistema conceptual de las diferentes disciplinas aplicadas. Esto puede ser una refutación de las hipótesis planteadas, lo que implicaría un rediseño de la estrategia y una nueva búsqueda de resultados, o un nuevo conocimiento.

Concebir el proceso de enseñanza aprendizaje según esta alternativa metodológica, debe propiciar que el futuro profesor adquiera hábitos y habilidades que le son inherentes, profundice en los aspectos conceptuales de las ciencias y la metodología científica, en un trabajo pedagógico interdisciplinario que debe favorecer su desempeño profesional en la práctica pedagógica de la realidad costarricense.

La aplicación sistemática del principio de la interdisciplinariedad debe ser inherente a la práctica pedagógica de la actual escuela costarricense, para esto es necesario que los nuevos planes de formación de los profesionales de la educación, las nuevas concepciones de trabajo docente estén permeadas de esta necesidad, por lo cual se hace evidente enfrentar este reto.

\section{Referencias bibliográficas}

Ander-Egg, E (1999). Interdisciplinaridad en educación. Editorial Magisterio Río de La Plata. Buenos Aires. Argentina.

Delors, J.(1996). La educación encierra un tesoro. Informe a la UNESCO de la Comisión Internacional sobre la Educación para el Siglo XXI. Ediciones UNESCO. París.

Escudero, J.(1990). Tendencias actuales en la investigación educativa: los desafíos de la investigáción crítica. Revista $\mathrm{Cu}$ rriculum. Universidad de La Laguna. Canarias. No. 2 
Fernández,M (1994). Las tareas de la profesión de enseñar. Editorial Escuela Española.Madrid.

Morin, E. (1998). Sobre la interdisciplinaridad. Boletín 2 del Centre Internacional de Recherches et Etudes Transdisiplinaires (CIRET). Recuperado el 07 de julio del 2009 de http://www.scribd.com/doc/7007366/Morin-Edgar-MorinSobre-La-Interdisciplinaridad 